\title{
Functional limitations after lateral column lengthening osteotomy of the calcaneus are associated with lower quality of life
}

\author{
*Corina Nüesch, $\mathrm{PhD}^{1,2}$, ORCID 0000-0002-7526-9536 \\ *Anna Katharina Schmid, MD candidate ${ }^{1}$ \\ S. Kimberly Byrnes, $\mathrm{MSc}^{1}$ \\ Philipp Krenn, MD ${ }^{1}$ \\ Monika Horisberger, $\mathrm{MD}^{1}$ \\ Annegret Mündermann, $\mathrm{PhD}^{1,2,3}$, ORCID 0000-0002-6472-1689 \\ André Leumann, $\mathrm{MD} / \mathrm{PhD}^{4}$
${ }^{1}$ Department of Orthopedics and Traumatology, University Hospital Basel, Basel, Switzerland
${ }^{2}$ Department of Biomedical Engineering, University of Basel, Basel, Switzerland
${ }^{3}$ Department of Clinical Research, University of Basel, Basel, Switzerland
${ }^{4}$ OrthoPraxis Leumann, Basel, Switzerland
${ }^{*} \mathrm{CN}$ and AKS share first authorship of this article

Original Article

International Orthopaedics

Doi: 10.1007/s00264-020-04526-9

Corresponding author: Prof. Dr. Annegret Mündermann

Department of Orthopedics and Traumatology

University Hospital Basel

Spitalstrasse 21

4031 Basel, Switzerland

Tel. +41 613285445

Email annegret.muendermann@unibas.ch

(C) International Orthopaedics, Springer, 2020, Attribution 4.0 International (CC BY 4.0) 


\begin{abstract}
Purpose

The purpose of this study was to quantify limitations in sagittal ankle range of motion (ROM) at least 2 years after lateral column lengthening osteotomy of the calcaneus (LLC) and their implications regarding quality of life.
\end{abstract}

\title{
Methods
}

Fifteen patients with a mean follow-up of $80 \pm 27$ months after LLC and 15 age-matched healthy persons participated in this study. Ankle joint complex ROM in plantarflexion and dorsiflexion was measured bilaterally using a goniometer and fluoroscopy (patients only). Quality of life was assessed using the short-form health questionnaire (SF36). Differences in ROM parameters (for the tibiotalar and subtalar joint) between sides (affected vs. unaffected) and between groups (patient vs. controls) and the relationship between ROM parameters and quality of life scores were assessed.

Results

ROM of the ankle joint complex on the affected side in patients was smaller than on the contralateral side (goniometer and fluoroscopy) and in healthy persons (goniometer; all $\mathrm{P}<.05)$. Among patients, SF36 total and pain scores, respectively, correlated with ROM of the subtalar joint (fluoroscopy; $\mathrm{R}=0.379, \mathrm{P}=0.039$ and $\mathrm{R}=0.537, \mathrm{P}=0.001$ ). Among patients and healthy persons, those with smaller dorsiflexion (goniometer) had lower quality of life scores.

\section{Conclusions}

The smaller sagittal ROM of the affected ankle joint complex compared to the contralateral foot and healthy controls was mainly explained by limitations in the tibiotalar joint. Because of its association with quality of life, ROM should be considered in the treatment and rehabilitation planning in patients who are candidates for LLC. 
Level of Evidence: Level III, retrospective cohort study

Key words: flat foot deformity; corrective surgery; range of motion; quality of life 


\section{Introduction}

Patients with adult acquired flatfoot deformity present with pain and functional limitations [1-3]. The reported prevalence is $3.3 \%$ in middle-aged women over 40 years of age [4]. Stage II is characterized by flexible hindfoot valgus, forefoot abduction and limited ability for single-leg heel rises $[5,6]$ and can be treated surgically by correcting the hindfoot position with calcaneal osteotomies. Several techniques such as medial sliding osteotomies of the tuber calcaneum or lateral column lengthening osteotomies of the calcaneus (LLC) have been described [7]. LLC procedures can be performed in different ways (Figure 1) - an osteotomy on the level of the sinus tarsi (Hintermann's osteotomy $[8,9]$ ), an osteotomy through the anterior process of the calcaneum (Evans procedure $[7,10]$ ) or as calcaneocuboid distraction arthrodesis [7].

Generally, good to excellent outcomes after LLC have been reported with significant improvements in pain $[9,11]$, clinical scores $[9,11-13]$, and quality of life scores $[11,13]$. Nevertheless, limitations in subtalar inversion/eversion range of motion (ROM) compared to the opposite foot have been reported in around $25 \%$ of the patients after Evans osteotomy or calcaneocuboid fusion [14], in more than $50 \%$ of patients after medial sliding calcaneal osteotomy [15], and with a reported $13^{\circ}$ smaller subtalar ROM compared to the opposite foot after LLC [12]. The motion in the different joints of the foot (i.e. tibiotalar, subtalar, talonavicular and calcaneocuboid joint) is coupled, and selective fusions of individual joints in cadavers affected the ROM of the other joints [16]. Moreover, a CT study [17] showed that both the tibiotalar and subtalar joint rotate between maximum dorsiflexion and maximum plantarflexion. Hence, the subtalar joint also contributes to sagittal ankle motion, and the contribution of the subtalar joint to plantarflexion/dorsiflexion ROM may differ between patients after anterior calcaneal distraction osteotomy $\left(8.6 \pm 6.4^{\circ}\right)$ and calcaneocuboid 
distraction osteotomy $\left(4.7 \pm 2.7^{\circ}\right)[17]$. Consequently, correcting the malalignment of the hindfoot through LLC might also influence the ROM of the ankle joint in plantarflexion and dorsiflexion, which would presumably be functionally important especially during more demanding ambulatory activities (e.g. for uphill walking).

The purpose of this study was to test the hypotheses that (i) the ROM of the ankle joint complex in the affected leg is smaller than in the contralateral limb and in healthy feet; (ii) the limitations in the ROM of the ankle joint complex are mainly explained by limitation in ROM of the subtalar joint; and (iii) patients with greater functional limitations have lower quality of life.

\section{Materials and Methods}

\section{Study cohort}

Fifteen patients (mean $\pm 1 \mathrm{SD}, 9$ female; age: $39.6 \pm 12.9$ years; body mass index (BMI): $26.3 \pm 3.5 \mathrm{~kg} / \mathrm{m}^{2}$ ) who had received LLC at least 2 years prior (follow-up: $80 \pm 27$ months) and 15 age-matched healthy persons ( 9 female; age: $40.2 \pm 13.7$ years; BMI: $23.0 \pm 2.8 \mathrm{~kg} / \mathrm{m}^{2}$ ) participated in this study. The surgeries performed were: isolated LLC (N=1), LLC with hallux valgus correction $(\mathrm{N}=2)$, LLC with autologous matrix induced chondrogenesis $(\mathrm{N}=4)$, LLC with refixation of os tibiale externum $(\mathrm{N}=2)$, LLC with osteotomy of the medial malleolus $(\mathrm{N}=1)$ and LLC with intervention of tendon or ligament tissue $(\mathrm{N}=5)$. The study was approved by the local ethics committee and all participants provided informed consent prior to participation. 


\section{Surgical procedure}

According to Hintermann et al. $[8,9]$, the sinus tarsi and the lateral calcaneus were exposed through a longitudinal incision at the base of the sinus tarsi (Figure 1). To protect cartilage and soft tissues such as the peroneal tendons and the interosseous ligaments, the LLC was directed between the two facets of the subtalar joint. The medial cortex was kept intact and based on the preoperative planning lateral lengthening was achieved under fluoroscopic control by securing an opening wedge with a pre-formed allograft with an axial cortical screw $(3.5 \mathrm{~mm})$.

\section{Clinical scores}

Subjective foot function was assessed using the German version of the Foot Function Index (FFI) [18] using the total score, and pain and disability subscores. The FFI ranges from 0 (no pain/disability) to 100 (maximal pain/disability). Quality of life was assessed using the shortform health questionnaire (SF36) with all subscores [19]. SF36 scores range from 0 (low) to 100 (high quality of life).

\section{ROM goniometer}

Ankle joint complex ROM in plantarflexion and dorsiflexion was measured bilaterally using a handheld goniometer. Participants lay supine on a treatment couch with the hip and knee flexed at $90^{\circ}$ and supported by the examiner with the foot in maximum dorsiflexion and maximum plantarflexion. In each position, the angle between the plantar surface of the foot and the long axis of the lower leg was measured. ROM was defined as the difference between maximum dorsiflexion and plantarflexion angle. 


\section{ROM fluoroscopy}

Maximum ankle ROM in plantarflexion and dorsiflexion were measured bilaterally using fluoroscopy in patients only (because of ethical concerns regarding radiation exposure in healthy persons) using the same positioning as with the handheld goniometer measurement. A C-arm image-intensifying fluoroscope (Arcadis Varic eco, Siemens AG, Erlangen; Germany) was aligned in true latero-medial view and used to capture fluoroscopic images of the ankle joint complex with the foot in maximum dorsiflexion and maximum plantarflexion.

On each fluoroscopic image, the line connecting the distal apices of the tibia was used as tibia reference line, the line connecting the geometric centers of two spheres fitted to the posterior and anterior aspect of the talus was used as talus reference line, and the line connecting the distal posterior and anterior apices of the calcaneus was used as calcaneus reference line (Figure 2). The angles between all reference lines were measured on all images. The ROM were defined as differences between maximum dorsiflexion and maximum plantarflexion images: ankle joint complex - tibia-calcaneus angle; tibiotalar joint - tibia-talus angle; and subtalar joint - talus-calcaneus angle. All fluoroscopic images were analyzed twice by the same tester (AKS) 6 months after the initial measurements to assess the repeatability of fluoroscopy-based ROM measurements with the tester being blinded to the results of the first measurements. The mean differences between repeated measurements of the different angles in the fluoroscopic images were $<1^{\circ}$ (P $>0.470$ for all). Coefficients of determination [20] were excellent for maximum dorsiflexion and plantarflexion of the ankle joint complex (0.920 and 0.913 , respectively) and good for maximum dorsiflexion and plantarflexion of the tibiotalar and subtalar joints (0.744 to 0.783$)$. Moreover, the fluoroscopic images were analyzed regarding osteoarthritic changes in the tibiotalar, subtalar, talonavicular, calcaneocuboid and Chopart joint using the Kellgren and Lawrence (KL) classification [21]. 


\section{Statistical analysis}

All statistical analyses were performed using MATLAB (The MathWorks Inc, version 2017b) and SPSS (IBM SPSS Statistics 22). Differences in parameters were detected using generalized mixed models with within-subject factor side (affected vs. unaffected) and between-subject factor group (patients vs. controls). Posthoc analyses were performed using paired (within subject) and independent (between groups) Student's t-tests and represented as 95\% confidence interval (CI). Stepwise multiple linear regression models were used to determine the contribution of the ROM in the tibiotalar and subtalar joints to the ROM of the ankle joint complex in patients using fluoroscopic measurements. Pearson's correlation coefficients were used to detect associations between parameters describing the ankle joint complex ROM and functional scores. The significance level for all statistical tests was set to $\alpha=0.05$.

\section{Results}

\section{Dorsiflexion/plantarflexion ROM}

On average, the ROM of the ankle joint complex on the affected side in patients was $7^{\circ}$ smaller than on the contralateral side $(\mathrm{P}=0.001)$ and $12^{\circ}$ smaller than in healthy persons $(\mathrm{P}=0.033$; goniometer measurements; Tables 1 and 2). Similarly, the ROM of the ankle joint complex measured using fluoroscopy was $11^{\circ}$ smaller on the affected than the contralateral side $(\mathrm{P}=0.001$; Tables 1 and 2$)$. The $\mathrm{ROM}$ of the tibiotalar joint on the affected side was $7^{\circ}$ smaller $(\mathrm{P}=0.018$; Tables 1 and 2$)$ and of the subtalar joint $2^{\circ}$ smaller than on the contralateral side $(\mathrm{P}=0.100)$. The latter difference was not statistically significant. 
Contribution of the subtalar joint to the ROM of the ankle joint complex

Linear regression analysis revealed that $55.7 \%$ of variability in ROM of the ankle joint complex measured using fluoroscopy in patients was explained by the variability in ROM of the tibiotalar joint $(\mathrm{R}=0.746 ; \mathrm{P}<0.001)$. Adding the $\mathrm{ROM}$ of the subtalar joint to the model explained most of the remaining variance resulting in an explained variance of $78.4 \%$ $(\mathrm{P}<0.001)$.

\section{Clinical scores/evaluation}

Patients had a higher total FFI with significantly higher pain and disability subscores $(\mathrm{P}<0.011$; Table 3). Quality of life was lower in patients than in healthy subjects: patients had lower SF36 total, physical function, social function, pain and general health subscores $(\mathrm{P}<0.028$; Table 3$)$. The other SF36 subscores did not differ between patients and healthy persons (Table 3).

Four patients had osteophyte formation and possible joint space narrowing (KL grade 2) in different foot joints of the surgically treated foot (subtalar, $\mathrm{N}=1$; talonavicular and Chopart, $\mathrm{N}=1$; calcaneocuboid and Chopart, $\mathrm{N}=1$; and tibiotalar, subtalar, calcaneocuboid, talonavicualar and Chopart, $\mathrm{N}=1$ ). All other joints in the affected feet and all joints in the contralateral feet had KL grades $<2$.

\section{Association between ankle ROM and clinical scores}

Among patients, SF36 total and pain subscores, respectively, correlated with ROM of the subtalar joint $(\mathrm{R}=0.379, \mathrm{P}=0.039$ and $\mathrm{R}=0.537, \mathrm{P}=0.001$, respectively). SF36 physical function correlated with ROM of the ankle joint complex $(\mathrm{R}=0.381, \mathrm{P}=0.038)$. Among patients and healthy persons, those with smaller dorsiflexion (goniometer) had lower quality 
of life: SF36 total, physical function and pain scores correlated with maximum dorsiflexion $(\mathrm{R}=0.285, \mathrm{P}=0.025 ; \mathrm{R}=0.378 . \mathrm{P}=0.002 ;$ and $\mathrm{R}=0.401, \mathrm{P}=0.001$, respectively). There was no significant association between FFI and ROM parameters.

\section{Discussion}

The purpose of this study was to quantify limitations in range of ankle motion in patients at least 2 years after LLC and their implications regarding quality of life. Our results confirmed our hypothesis that the ROM of the ankle joint complex in the affected leg is smaller than in the contralateral limb and in healthy feet and that patients with greater functional limitations have lower quality of life. In contrast to our hypothesis, limitations in ROM of the subtalar joint explained the limitations in the ROM of the ankle to a lesser extent than did the limitations in ROM of the tibiotalar joint.

\section{ROM and clinical scores/evaluation}

While previous studies have reported limited frontal plane subtalar ROM compared to the contralateral foot in patients after LLC $[12,14,15]$, we focused on the sagittal plane of the ankle joint. While our results are not directly comparable with existing literature, they suggest that the sagittal ankle joint motion is also affected by LLC especially because of a limited amount of dorsiflexion (measured with goniometer).

Patients reported lower quality of life than healthy controls manifested in lower SF36 total, physical function, social function, pain and general health subscores. However, scores in our study were higher than those in other studies reporting SF36 scores as measure of quality of life after LLC procedures. For instance, the total SF36 score in our study was higher than in patients with an average follow-up of 40 months after Hintermann's osteotomy $(79.4 \pm 13.8$ 
vs. $69.4 \pm 20.5$ ) [13]. Similarly, in our study subscores for physical function, mental health, and physical health were on average above 74 , while another study reported values of around 60 in patients without lateral foot pain after Evans type LLC and of around 30 (physical function and health) and 55 (mental health), respectively, in patients with lateral foot pain [22]. Moreover, while patients in our study had a lower FFI than the healthy controls, the average score was comparable to the score of patients assessed 2 years after calcaneocuboid distraction arthrodesis (19.2 \pm 17.7 vs. $22.1 \pm 15.4)$ [23]. This indicates generally a good outcome and quality of life in our patients compared to other patient populations that underwent a similar surgery.

Four patients in our study had osteoarthritic changes of KL grade 2 in their affected foot. We observed osteoarthritic changes in the calcaneocuboid joint in two patients (13\%), which is more frequent than what has been reported in another study after Hintermann's LLC $(2.8 \%$ $(1 / 36))$ [13]. In addition to different x-ray techniques (conventional weight bearing x-ray vs. fluoroscopy), the longer follow-up in our study ( $80 \mathrm{vs.} 40$ months) could contribute to this different result. Interestingly, none of the patients had osteoarthritic changes in the contralateral foot. Whether these changes may be caused by the surgical intervention or if they would have naturally developed because of the deformity cannot be elucidated in this study.

\section{Contribution of the subtalar joint}

The contribution of the subtalar joint to the plantarflexion/dorsiflexion ROM was small confirming previous findings for both subtalar and tibiolatar ROM in a CT study in patients after anterior calcaneal distraction osteotomy and calcaneocuboid distraction arthrodesis [17]. While the ROM of the subtalar joint contributed to explaining the variability of the ROM of 
the ankle joint complex, the majority was explained by the ROM of the tibiotalar joint. Hence, our second hypothesis that the limitations in subtalar joint ROM cause the limitations in the ROM of the ankle joint complex was not supported by our data. Interestingly, the ROM of the subtalar joint was associated with the SF36 scores (total score and pain subscore). In vivo CT and MRI measurements in healthy persons have shown that the subtalar plantarflexion/dorsiflexion ROM varies largely between subjects [24,25]. Hence, our results imply that patients might be sensitive to limitations in subtalar motion - even in the sagittal plane - and that this might impact pain and quality of life.

Across patients and healthy persons, maximum dorsiflexion, but not dorsiflexion/plantarflexion ROM, contributed to SF36 total, physical function and pain scores. This result suggests that quality of life is primarily affected by limitation in ankle dorsiflexion (rather than ROM) and that stretching and maintaining/regaining dorsiflexion is important during rehabilitation and daily life in this patient population. It is possible that ROM was already limited before surgery in some patients. The associations between ROM and quality of life reported here suggest that preoperative ROM could potentially influence the success of LLC and play a role in the long-term outcome in these patients.

\section{Limitations}

This study cohort of fifteen patients is a rather small cohort, and most patients had additional surgical procedures to correct foot alignment. Hence, the results of this study may be specific to this cohort and care should be taken in generalizing these results to other cohorts.

Moreover, preoperative fluoroscopic ROM measurements were not available and hence, it is not known whether some of the limitations already existed preoperatively. Due to ethical reasons, no fluoroscopy measurements were performed in healthy persons, and thus a 
comparison of the ROM was only possible with the contralateral side or for values measured with a goniometer. Nevertheless, this study presents detailed data on the ROM in patients who had undergone LLC surgery with a longer follow-up than previous studies (at least 2 years and on average more than 6 years) and their relation to quality of life.

\section{Conclusion}

In conclusion, this study showed that the ROM of the ankle joint complex was smaller in the affected foot compared to the contralateral foot and healthy controls and that this limitation was mainly explained by limitations in the tibiotalar joint. Because of its association with quality of life, ROM should be considered in the treatment and rehabilitation planning in patients who are candidates for LLC.

\section{Conflict of interest}

There were no conflicts of interest.

\section{Ethical approval}

All procedures performed in studies involving human participants were in accordance with the ethical standards of the institutional and/or national research committee and with the 1964 Helsinki declaration and its later amendments or comparable ethical standards.

\section{References}

1. Kohls-Gatzoulis J, Angel JC, Singh D, Haddad F, Livingstone J, Berry G (2004) Tibialis posterior dysfunction: a common and treatable cause of adult acquired flatfoot. Bmj 329 (7478):1328-1333 
2. Beeson P (2014) Posterior tibial tendinopathy: what are the risk factors? Journal of the American Podiatric Medical Association 104 (5):455-467

3. Smyth NA, Aiyer AA, Kaplan JR, Carmody CA, Kadakia AR (2017) Adult-acquired flatfoot deformity. European Journal of Orthopaedic Surgery \& Traumatology 27 (4):433439. doi:10.1007/s00590-017-1945-5

4. Kohls-Gatzoulis J, Woods B, Angel J, Singh D (2009) The prevalence of symptomatic posterior tibialis tendon dysfunction in women over the age of 40 in England. Foot and Ankle Surgery $15(2): 75-81$

5. Johnson KA, Strom DE (1989) Tibialis posterior tendon dysfunction. Clinical Orthopaedics and Related Research (1976-2007) 239:196-206

6. Fuhrmann RA, Trommer T, Venbrocks RA (2005) [The acquired buckling-flatfoot. A foot deformity due to obesity?]. Orthopade 34 (7):682-689. doi:10.1007/s00132-005-0823-8

7. Mosier-Laclair S, Pomeroy G, Manoli A (2001) Operative treatment of the difficult stage 2 adult acquired flatfoot deformity. Foot and ankle clinics 6 (1):95-119

8. Hintermann B, Valderrabano V (2003) Lateral column lengthening by calcaneal osteotomy. Techniques in Foot \& Ankle Surgery 2 (2):84-90

9. Hintermann B, Valderrabano V, Kundert H-P (1999) Lengthening of the lateral column and reconstruction of the medial soft tissue for treatment of acquired flatfoot deformity associated with insufficiency of the posterior tibial tendon. Foot $\&$ ankle international 20 (10):622-629

10. Evans D (1975) Calcaneo-valgus deformity. The Journal of bone and joint surgery British volume $57(3): 270-278$

11. Kou JX, Balasubramaniam M, Kippe M, Fortin PT (2012) Functional results of posterior tibial tendon reconstruction, calcaneal ostetomy, and gastrocnemius recession. Foot $\&$ ankle international 33 (7):602-611 
12. Valderrabano V, Hintermann B, Wischer T, Fuhr P, Dick W (2004) Recovery of the posterior tibial muscle after late reconstruction following tendon rupture. Foot \& Ankle International 25 (2):85-95

13. Ettinger S, Mattinger T, Stukenborg-Colsman C, Yao D, Claassen L, Daniilidis K, Plaass C (2019) Outcomes of Evans Versus Hintermann Calcaneal Lengthening Osteotomy for Flexible Flatfoot. Foot \& ankle international 40 (6):661-671

14. Thomas RL, Wells BC, Garrison RL, Prada SA (2001) Preliminary results comparing two methods of lateral column lengthening. Foot \& ankle international 22 (2):107-119

15. Myerson MS, Badekas A, Schon LC (2004) Treatment of stage II posterior tibial tendon deficiency with flexor digitorum longus tendon transfer and calcaneal osteotomy. Foot \& ankle international 25 (7):445-450

16. Wülker N, Stukenborg C, Savory KM, Alfke D (2000) Hindfoot motion after isolated and combined arthrodeses: measurements in anatomic specimens. Foot \& Ankle International 21 (11):921-927

17. Beimers L, Louwerens JW, Tuijthof GJM, Jonges R, Niek van Dijk C, Blankevoort L (2012) CT measurement of range of motion of ankle and subtalar joints following two lateral column lengthening procedures. Foot \& ankle international 33 (5):386-393

18. Naal FD, Impellizzeri FM, Huber M, Rippstein PF (2008) Cross-cultural adaptation and validation of the Foot Function Index for use in German-speaking patients with foot complaints. Foot \& Ankle International 29 (12):1222-1231

19. Ware JE, Sherbourne CD (1992) The MOS 36-Item Short-Form Health Survey (SF-36):

I. Conceptual Framework and Item Selection. Medical Care 30 (6):473-483

20. Fleiss J (1986) The Design and analysis of clinical experiments. John Wiley \& Sons, NewYork 
21. Kellgren J, Lawrence J (1957) Radiological assessment of osteo-arthrosis. Annals of the rheumatic diseases $16(4): 494$

22. Ellis SJ, Joseph CY, Johnson AH, Elliott A, O'Malley M, Deland J (2010) Plantar pressures in patients with and without lateral foot pain after lateral column lengthening. The Journal of Bone \& Joint Surgery 92 (1):81-91

23. van der Krans A, Louwerens JWK, Anderson P (2006) Adult acquired flexible flatfoot, treated by calcaneo-cuboid distraction arthrodesis, posterior tibial tendon augmentation, and percutaneous Achilles tendon lengthening: A prospective outcome study of 20 patients. Acta Orthopaedica 77 (1):156-163. doi:10.1080/17453670610045858

24. Sheehan FT, Seisler AR, Siegel KL (2007) In vivo talocrural and subtalar kinematics: a non-invasive 3D dynamic MRI study. Foot \& ankle international 28 (3):323-335

25. Beimers L, Maria Tuijthof GJ, Blankevoort L, Jonges R, Maas M, van Dijk CN (2008) In-vivo range of motion of the subtalar joint using computed tomography. Journal of Biomechanics 41 (7):1390-1397. doi:https://doi.org/10.1016/i.jbiomech.2008.02.020 


\section{Figures}
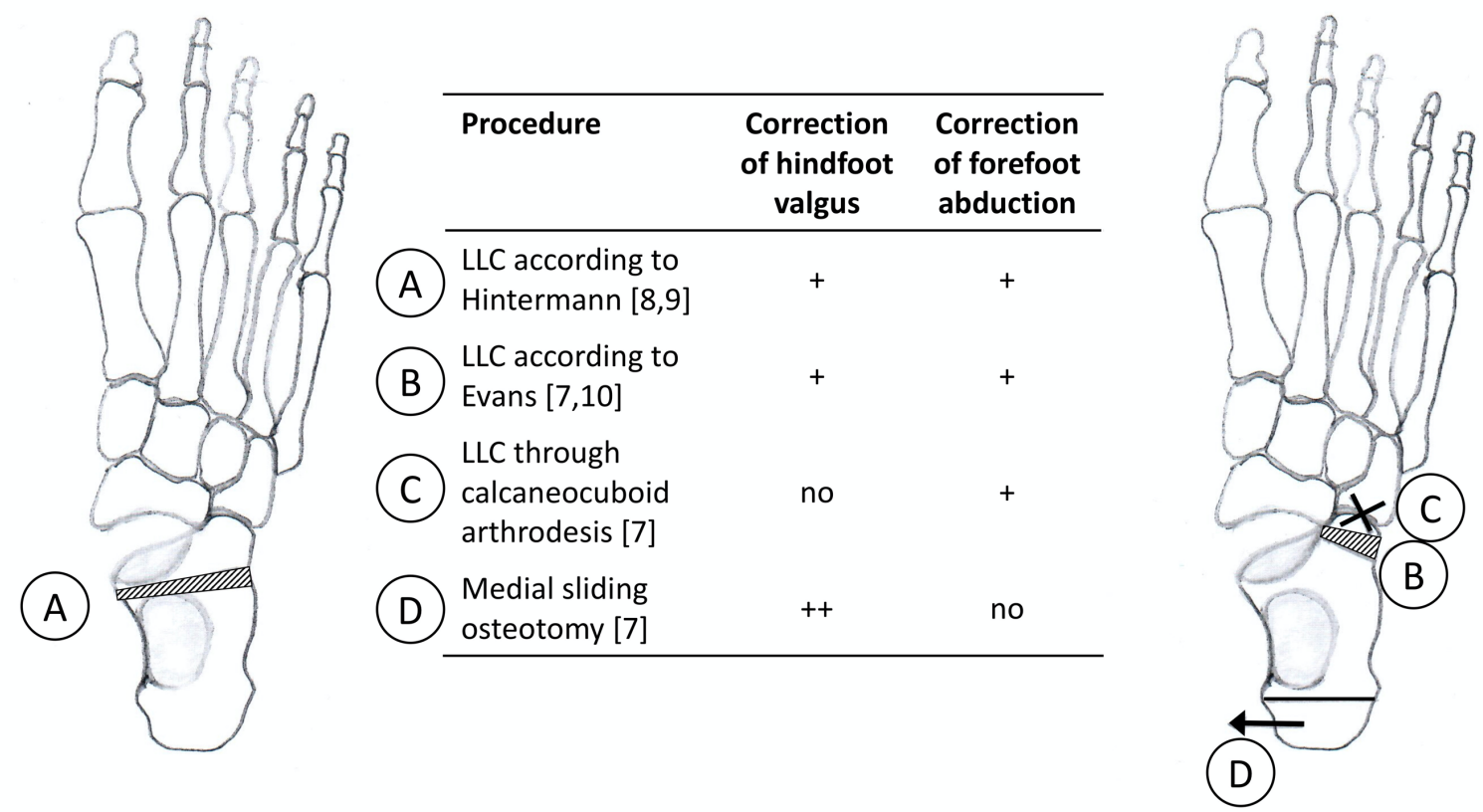

Figure 1: Overview of different surgical procedures used to correct the adult acquired

flatfoot deformity and their correction potential on the hindfoot valgus and forefoot abduction (no-little potential; +-some potential; ++-strong potential). The procedure that was performed in our study is depicted on the left (A), alternatives are depicted on the right (BD). 


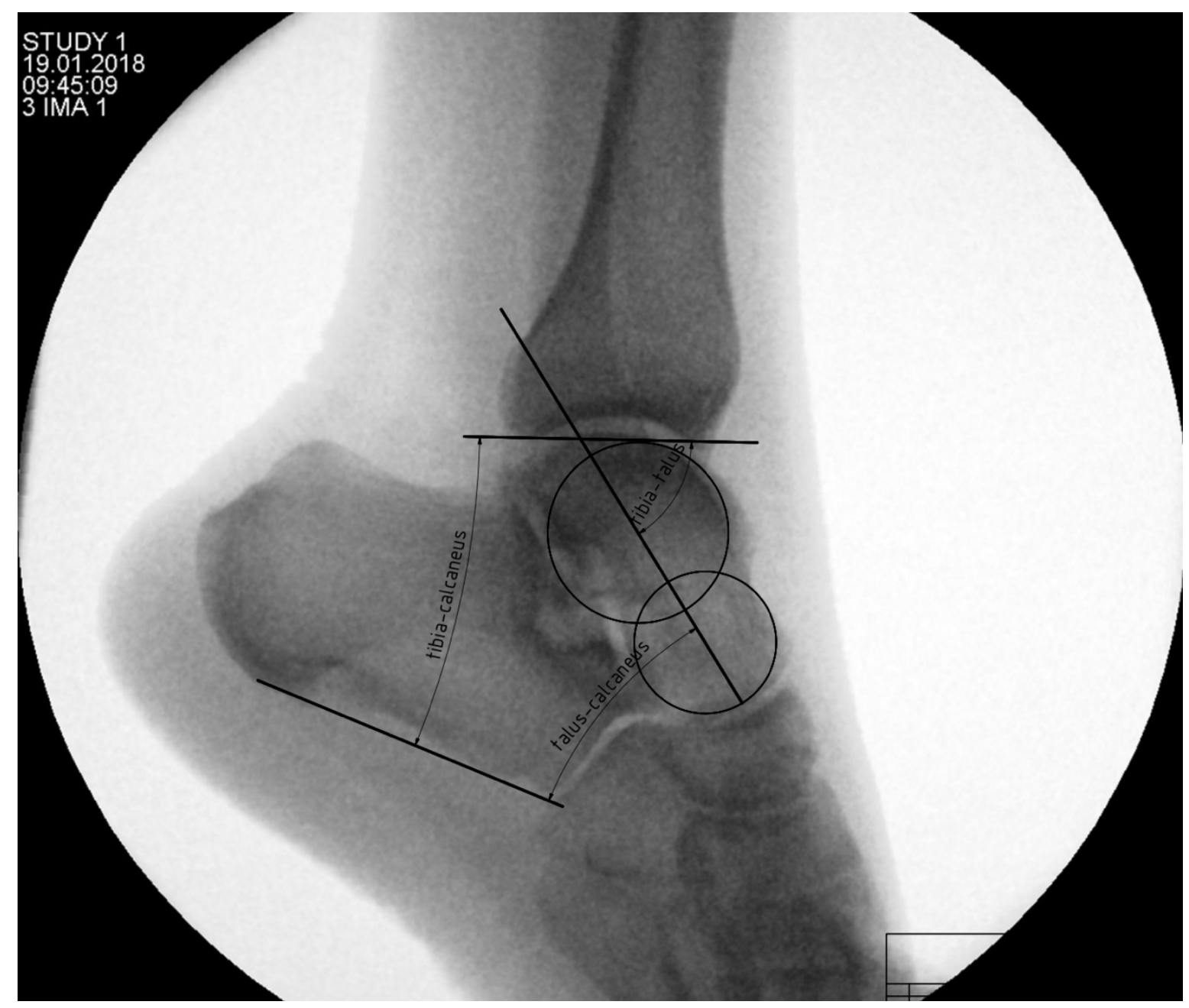

Figure 2: Illustration of joint angles measured using single sagittal plane fluoroscopy. 


\section{Tables}

Table 1. Mean (1 standard deviation) flexibility for the affected and contralateral limbs in patients and left and right limbs in healthy subjects.

\begin{tabular}{|c|c|c|c|c|c|c|}
\hline \multirow{2}{*}{ Parameter } & \multicolumn{2}{|c|}{ Patients $(N=15)$} & \multicolumn{2}{|c|}{ Healthy subjects $(N=15)$} & \multicolumn{2}{|c|}{$P$-value ANOVA } \\
\hline & Affected & Contralateral & Left & Right & Side & Side $\times$ group \\
\hline \multicolumn{7}{|l|}{ Flexibility (goniometer) } \\
\hline $\begin{array}{l}\text { ROM ankle joint complex } \\
\left({ }^{\circ}\right)\end{array}$ & $65.2(7.6)$ & $72.6(9.8)$ & $75.9(17.0)$ & $77.3(16.4)$ & $<0.001$ & 0.005 \\
\hline Maximum dorsiflexion $\left({ }^{\circ}\right)$ & $9.7(3.8)$ & $14.5(4.1)$ & $17.5(6.3)$ & $18.9(6.6)$ & 0.001 & 0.039 \\
\hline $\begin{array}{l}\text { Maximum plantarflexion } \\
\left({ }^{\circ}\right)\end{array}$ & $55.5(7.2)$ & $58.1(8.6)$ & $58.4(17.8)$ & $58.5(16.7)$ & 0.139 & 0.159 \\
\hline \multicolumn{7}{|l|}{ Flexibility (fluoroscopy) } \\
\hline $\begin{array}{l}\text { ROM ankle joint complex } \\
\left({ }^{\circ}\right)\end{array}$ & $52.8(10.2)$ & $63.8(9.2)$ & n.a. & n.a. & 0.001 & n.a. \\
\hline ROM tibiotalar joint $\left({ }^{\circ}\right)$ & $47.8(7.7)$ & $54.4(9.5)$ & n.a. & n.a. & 0.018 & n.a. \\
\hline ROM subtalar joint $\left(^{\circ}\right)$ & $6.1(3.9)$ & $8.6(4.0)$ & n.a. & n.a. & 0.100 & n.a. \\
\hline
\end{tabular}

ROM - range of motion; n.a. - not available 
Table 2. 95\% confidence intervals of the difference between affected and contralateral limb in patients (paired comparison) and between the affected and contralateral limbs in patients and left and right limbs in healthy subjects (unpaired comparison), respectively.

\begin{tabular}{|c|c|c|c|}
\hline Parameters & $\begin{array}{c}\text { Affected- } \\
\text { contralateral }\end{array}$ & $\begin{array}{l}\text { Affected- } \\
\text { healthy left }\end{array}$ & $\begin{array}{c}\text { Contralateral- } \\
\text { healthy right }\end{array}$ \\
\hline \multicolumn{4}{|l|}{ Flexibility (goniometer) } \\
\hline ROM ankle joint complex $\left({ }^{\circ}\right)$ & {$[-11.3 ;-3.5]$} & {$[-20.5 ;-0.93]$} & {$[-14.7 ; 5.4]$} \\
\hline Maximum dorsiflexion $\left({ }^{\circ}\right)$ & {$[-8.1 ;-1.6]$} & {$[-11.7 ;-3.9]$} & {$[-8.3 ;-0.1]$} \\
\hline Maximum plantarflexion $\left({ }^{\circ}\right)$ & {$[-5.3 ; 0.2]$} & {$[-13.0 ; 7.2]$} & {$[-10.3 ; 9.4]$} \\
\hline \multicolumn{4}{|l|}{ Flexibility (fluoroscopy) } \\
\hline ROM ankle joint complex $\left({ }^{\circ}\right)$ & {$[-8.6 ;-2.9]$} & n.a. & n.a. \\
\hline ROM tibiotalar joint $\left({ }^{\circ}\right)$ & {$[-11.9 ;-1.3]$} & n.a. & n.a. \\
\hline ROM subtalar joint $\left(^{\circ}\right)$ & {$[-5.6 ; 0.6]$} & n.a. & n.a. \\
\hline
\end{tabular}

ROM - range of motion; n.a. - not available 
Table 3. Mean (1 standard deviation) functional scores for patients and healthy subjects.

\begin{tabular}{|c|c|c|c|c|}
\hline Parameter & $\begin{array}{l}\text { Patients } \\
(N=15)\end{array}$ & $\begin{array}{l}\text { Healthy subjects } \\
(N=15)\end{array}$ & $\begin{array}{c}P \text {-value } \\
\text { t-test }\end{array}$ & $\begin{array}{l}\text { 95\% CI patients- } \\
\text { healthy subjects }\end{array}$ \\
\hline \multicolumn{5}{|l|}{ Foot function index } \\
\hline Pain scale & $17.6(19.3)$ & $2.3(3.7)$ & 0.009 & {$[4.5 ; 26.1]$} \\
\hline Disability scale & $20.6(22.2)$ & $2.0(5.2)$ & 0.011 & {$[5.0 ; 32.2]$} \\
\hline Total & $18.8(18.3)$ & $2.1(3.3)$ & 0.002 & {$[6.4 ; 26.9]$} \\
\hline \multicolumn{5}{|l|}{ Quality of life - SF36 } \\
\hline Total & $79.4(13.8)$ & $88.8(4.7)$ & 0.016 & {$[-16.8 ;-1.9]$} \\
\hline Physical function & $82.9(20.9)$ & $88.8(3.9)$ & 0.006 & {$[-26.7 ;-5.0]$} \\
\hline Role of physical function & $87.1(25.6)$ & $95.7(9.9)$ & 0.221 & {$[-22.7 ; 5.5]$} \\
\hline Role of emotional function & $95.0(7.6)$ & $91.1(17.6)$ & 0.441 & {$[-6.2 ; 13.9]$} \\
\hline Vitality & $64.6(15.8)$ & $68.0(6.8)$ & 0.439 & {$[-12.2 ; 5.4]$} \\
\hline Mental health & $82.3(9.4)$ & $79.7(8.3)$ & 0.412 & {$[-3.9 ; 9.1]$} \\
\hline Social function & $91.7(13.1)$ & $100.0(0.0)$ & 0.016 & {$[-15.0 ;-1.7]$} \\
\hline Pain & $82.0(25.4)$ & $96.7(6.5)$ & 0.001 & {$[-38.2 ;-11.3]$} \\
\hline General health & $74.2(22.4)$ & $88.8(9.8)$ & 0.028 & {$[-27.5 ;-1.7]$} \\
\hline
\end{tabular}

CI - confidence interval; SF36 - Short Form Health 36 